\title{
Research and Application of Thickness Control Strategies in Steel Plate Rolling
}

\author{
Fei Zhang ${ }^{1, *}$, Yongjun Zhang ${ }^{1}$, Jianxin Hou ${ }^{1}$ and Ling Huang ${ }^{2}$

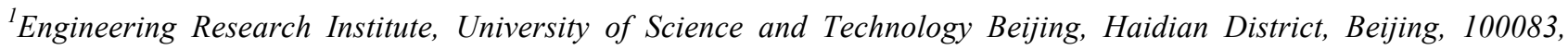 \\ China \\ ${ }^{2}$ College of Electric and Information Engineering, Guangxi University of Science and Technology, Liuzhou, 545006, \\ China
}

\begin{abstract}
Thickness accuracy of plate production depends on Automatic Gauge Control (AGC) in the plate mill area. A typical modern thickness control system design and implementation of a steel plate mill is discussed together with the impact on the design of process models, available measurements, available control actuators and material properties. A highlevel software design approach provides the ability to make quick and accurate modifications to both control loops and tuning constants. Control loop strategies and theoretical concepts of steel plate rolling are discussed. Performance results achieved during the startup of a steel plate mill are included.
\end{abstract}

Keywords: Automatic gauge control, Steel plate mill, Thickness control, Roll thermal expansion, Mill modulus control.

\section{INTRODUCTION}

The schematic diagram of a plate mill is shown in Fig. (1). The mill stand consists of a mill housing containing two cylindrical work rolls, which perform the reduction, and a pair of larger backup rolls, which support them. Gap control includes the control for the top mounted variable speed synchronous motor driven no-load screwdowns and a bottom mounted servo controlled hydraulic cylinders [1]. These two actuators are controlled as required to support roll change, plate rolling and mill testing.

The thickness of the steel is controlled by dynamic roll positioning. Because the reduction of hot steel in the roll bite is sensitive to mill speed changes as well as temperature and hardness changes in strip entry, the gauge control must be able to control to the desired thickness of strip in the face of these disturbances. This control involves the careful coordination of a number of strategies. The rolls are placed prior to entry bar of the mill. It involves predictive models of the steel-rolling process, which will give accurate roll-position settings. To produce output strip of the desired thickness, the roll position is then altered during rolling of the bar. This dynamic roll positioning during rolling of the strip is referred to as automatic gauge control (AGC). The results of rolling each strip are further analyzed and used as adaptive corrections to the predictive models [2].

The success of the thickness control depends on the ability of these various facets of the control to blend in a cohesive system. This blending is particularly evident in the successful development of the absolute, or predicted, mode of automatic gauge control. This mode of control involves an intimate connection between the traditional mill setup and AGC functions. It can prove that a structured system design approach is necessary to ensure the proper integration of the various components of the thickness control [3].

\section{THICKNESS CONTROL OVERVIEW}

The various components of the thickness control in the setup and AGC functions take the form of accurate and responsive control loops. A block diagram of these control loops is shown in Fig. (2). Two major sources of disturbances that must be eliminated in the control of strip thickness are roll-gap errors and strip-hardness errors. The roll gap is modeled by a number of open-loop models, which are calibrated using mill tests. Residual roll gap errors are isolated using X-ray thickness measurements and the principle of mass flow and are used as adaptive feedback for the roll-gap models. These errors are usually small, and it is because the roll gap can be modeled accurately that the absolute mode of AGC can operate so successfully.

Strip hardness and deformation characteristics are also modeled for the numerous grades of steel and rolling conditions. Errors in these models are isolated using roll-force predictions during the adaptive feedback process. Any remaining steady-state error is removed by the mill vernier feedback, which compares the desired thickness with the actual thickness. These bar-to-bar control loops work in conjunction with the in-bar automatic gauge control. 


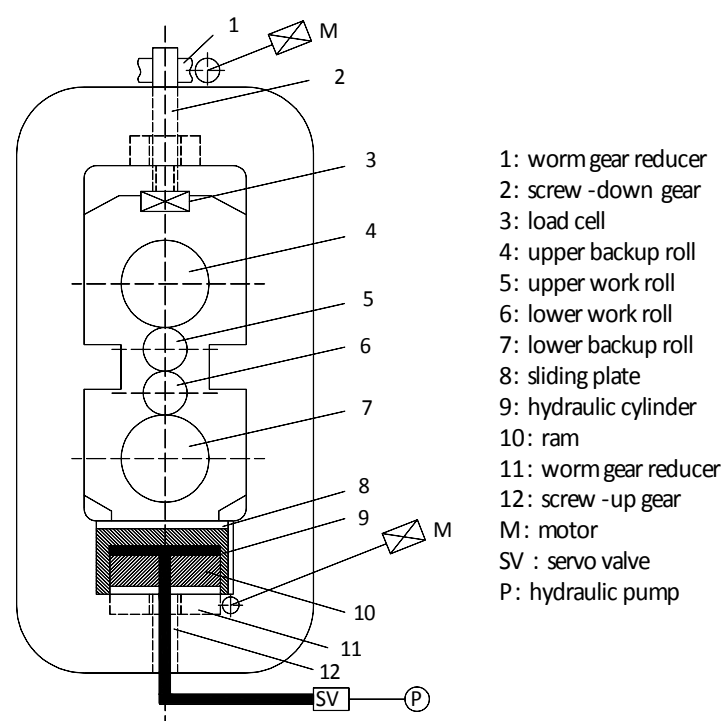

Fig. (1). Schematic Diagram of a Plate Mill.

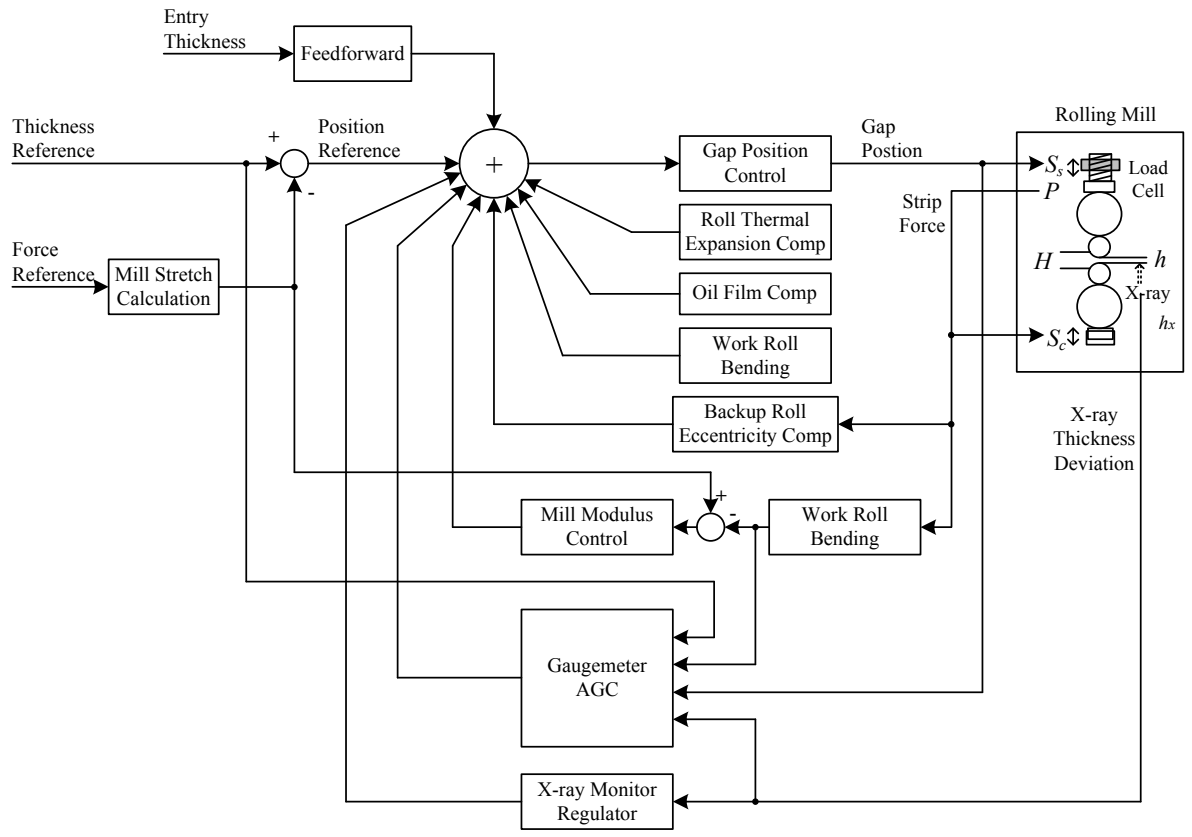

Fig. (2). AGC Block Diagram.

Automatic gauge control uses two methods to measure strip thickness. The first uses the roll position and force measurements at the rolling mill stand as the basic one. Strip thickness differs from roll position because of various mill housing deflections and roll deformations, which are compositely known as "mill stretch". The total roll force of the stand reacts to this mill stretch but also reacts to changes in the mill stand. Therefore, while roll position and force measurements offer the potential of an immediate measure of strip thickness at each stand, certain prerequisites must be met. Because roll force is one indicator of mill stretch, it is necessary to know how to extract it and convert it to mill stretch by an accurate transfer function. The control of strip thickness using roll position and force measurements is known as
Gaugemeter, or BISRA, AGC, named after the British Iron and Steel Research Association. In practice, different control goal requires different mill modulus in the manufacture process of hot rolling mill $[4,5]$. AGC can compensate elastic deformation of the rolling mill to a certain extent when rolling force fluctuates. In other words, the mill modulus can be controlled. So, there is a concept of the mill modulus control (MMC) to be brought forward. According to different requirement of rolling technology, different equivalent mill modulus can be obtained while changing adjustment coefficients in the AGC system. MMC also uses the Gaugemeter equation to estimate stand exit strip thickness changes.

The second measurement of strip thickness is provided by a thickness gauge at the exit of the plate mill. This device 


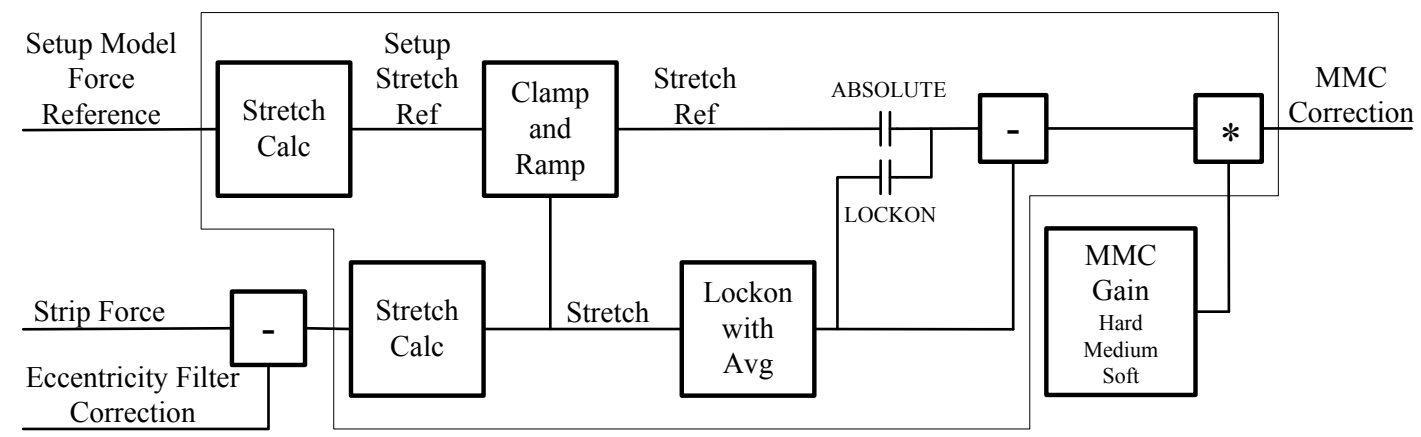

Fig. (3). Simplified MMC Block Diagram.

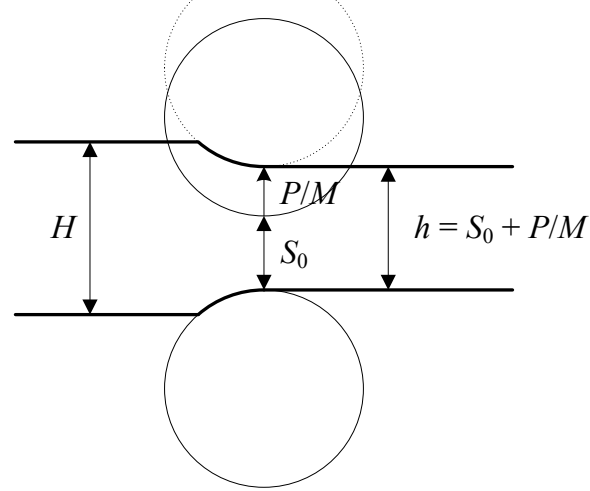

Fig. (4). Gaugemeter Equation.

accurately measures the strip thickness in a distance from the mill and therefore gives a delayed measurement of the strip thickness. This means that the control that uses the thickness gauge must carefully account for this delay. The control of strip thickness using thickness gauge measurements is known as monitor control. By using the measured thickness, Feedforward AGC is provided to help compensation for known incoming product variations. Feedforward AGC assists MMC and Gaugemeter as they cannot completely correct gauge due to factors such as window friction and load cell hysteresis.

\subsection{Mill Modulus Control (MMC)}

MMC modifies gap position to maintain a constant delivery strip thickness from the stand. MMC uses the Gaugemeter equation to estimate stand exit strip thickness changes. MMC control provides an absolute and a lock-on mode. Automatic gauge control can be deselected (including MMC) for the stand. While the Gaugemeter equation is used for both modes of operation, the different modes determine how the reference is generated and the turn on time of the function. After installation the control is intended to operate in absolute mode so there is no operator selection.

The force reference from the setup model is used to calculate the setup stretch reference. Strip force is used to calculate the stretch error. The setup stretch reference and stretch are used by the clamp and ramp function to determine the absolute stretch reference. If absolute is selected and after the absolute time delay has expired, the absolute stretch reference is used and the control becomes active. If lock-on is selected and after the lock-on time delay has expired, the calculated stretch is used as the stretch reference and the control becomes active. The stretch error is calculated so that if the stretch error becomes more positive, the gap will close. The amount of correction that is applied is controlled by the MMC gain. Gains are provided for three ranges of strip hardness (hard, medium and soft). Fig. (3) is a simplified block diagram of the MMC function.

\subsection{Gaugemeter Control (GM)}

Gaugemeter control uses the Gaugemeter equation $(\mathrm{h}=$ $\mathrm{S} 0+\mathrm{P} / \mathrm{M}$, see Fig. 4) to estimate exit strip thickness at each stand [6]. GM uses the calculated strip thickness as the feedback to maintain the desired delivery strip thickness from the stand by modifying gap position. GM is a slower outer control loop that tries to achieve a desired strip thickness in contrast to MMC that is the high speed inner control loop that tries to instantly correct for mill stretch changes. After installation the control is intended to operate in Absolute mode so there is no operator selection. GM control provides an absolute and a lock-on mode. The mode of control is selected for the stand. While the Gaugemeter equation is used for both modes of operation, the different modes determine how the reference is generated and the turn on time of the function. 


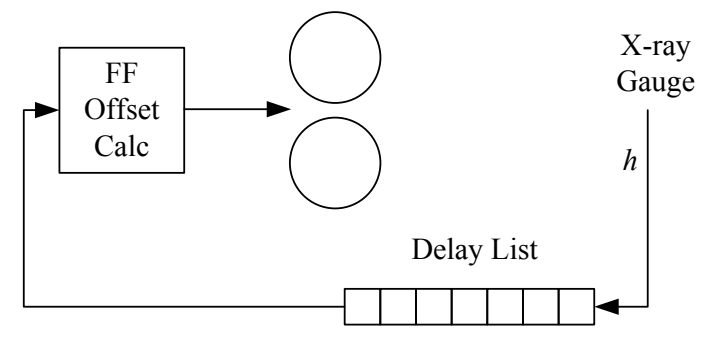

Fig. (5). Feedforward Function Overview.

GM first calculates the exit strip thickness by the Gaugemeter method using gap position, gap compensations and calculated mill stretch. A lock-on thickness is calculated for lock-on GM by multiplying a Lock-on Stretch Gain times the calculated mill stretch. Similarly, an absolute thickness is calculated for absolute GM using an Absolute Stretch Gain. For absolute GM, a portion of the calculated setup stretch is also added for the amount of stretch ignored by the Absolute Stretch Gain. If the Smith Method Monitor is active, that offset will also be included in the absolute thickness feedback.

A proportional plus integral control is applied to the GM error. The resulting strip thickness correction is multiplied by a transfer function that calculates the appropriate gap correction to achieve the desired strip thickness change. The selected GM Stretch Gain (Absolute or Lock-on) and the MMC Stretch Gain, as well as the Mill and Strip Modulus, affect the transfer function [7].

\subsection{X-RAY Monitor}

The X-ray Monitor regulates mill exit thickness using strip thickness deviation feedback measured by an X-ray gauge. The measured thickness deviation is integrated and the result is applied as a gap correction to the gap control for the stand. X-ray Monitor defaults to Absolute and will produce gap corrections to make the strip thickness equal the Xray gauge target thickness (zero deviation). However, the control can direct the X-ray Monitor to re-target to the measured thickness with a Auto Lock-on feature to help prevent cobbles.

The reference for the regulator is normally zero deviation but will become a non-zero deviation if Auto Lock-on occurs because of an excessive initial error. The feedback is the selected X-ray gauge measured deviation. The gauge error is calculated as the difference between the deviation reference and the deviation feedback. The gauge error is used to calculate the correction.

\subsection{Feed for Ward}

Feedforward AGC is provided to help compensate for known incoming product variations. Feedforward assists MMC and Gaugemeter as they cannot completely correct gauge due to factors such as window friction and load cell hysteresis.
Feedforward AGC measures an entry thickness. Since there is only one X-ray gauge (exit side of the mill) Feedforward is only available on reverse passes. The entry thickness is recorded in a list and tracked as the strip moves to the mill. As a segment of strip approaches the stand, the thickness is removed from the list and used to calculate a gap correction designed to enable MMC to remove the entire deviation.

Feedforward AGC performs the tasks listed below and illustrated in Fig. (5).

The Feedforward AGC gauge correction is then computed to be the amount of expected delivery gauge error from the expected equivalent entry gauge change.

The thickness value is removed from the delay list slightly before it arrives at the stand to allow the position regulator time to respond.

\section{GAP COMPENSATIONS}

Gap Compensations are open loop corrections to the Gap Position Regulator for known effects that change the effective roll gap opening but are not directly measured by the gap position transducers. Gap position transducers measure the amount of cylinder (and/or screw) extension. Although the measurements are very accurate, between position measurements and the actual gap opening are the backup roll chock and bearings, the backup roll diameter and the work roll diameter. When force is applied, the mill housing stretches, the backup roll necks bend, the roll surfaces flatten at the points of contact, the oil in the cylinder compresses and the backup roll moves off center in its bearing. As the mill stand speed increases the backup roll will become more centered in its bearing. As the rolls heat up they expand and their diameters increase. As the rolls wear their diameters decrease. The amount the rolls bend changes as roll bending force changes. The centerline gap changes as the side shift position changes. Force measurements (load cells) drift. In addition, the strip thickness changes when strip tension changes.

Some of these effects are predictable and so an open loop estimate of the effect on the actual roll gap opening is calculated and used to establish the roll gap position. The mill zeroing procedure accounts for all the factors under the specific zeroing conditions of speed and force. The open loop 
calculations attempt to take care of the changes from the zeroing conditions. In general, factors that change slowly like roll wear and long term roll heating can be calculated by the setup models. Factors that change quickly are calculated by AGC functions [8].

The stretch of the mill housing and the stack deflection (roll chock dimension changes with force and roll flattening) are corrected by the MMC AGC function. The Backup Roll Eccentricity function compensates for backup roll diameter variations during a revolution. Compensation for oil compression in the gap cylinders and manual load cell zeroing are provided by the gap control function. All other effects that can be predicted by open loop calculations are grouped into the gap compensations AGC function.

\subsection{Oil Film Compensation}

The backup rolls settle to the outer side of their bearings at low speeds, but as the rotation of the backup roll increases, the bearing oil is pulled evenly around the roll, causing the roll to center itself. The speed effect on roll gap is to decrease roll opening as mill speed increases. The magnitude of this effect decreases as roll force increases. The gap position sensors do not detect the change in roll gap opening. This change can be significant in oil type bearings and can cause large errors in gage control if not properly compensated. Roller bearings may not require compensation.

This compensation provides an offset to the Gap Position regulator to maintain a constant effective gap as mill speed changes. The effect on the roll gap is separated into a speed effect and a force effect. The speed effect determines the correction to roll gap position with the roll separating force equal to zeroing force. The speed effect is zero at zero speed. As speed increases the gap will be opened. The force effect determines a per unit modifier which relates the correction at other forces to the correction required for zeroing force. The per-unit effect is 1.0 at zeroing force. As force increases the amount of correction must be reduced.

Curves of the speed effect and force effect are stored as arrays. Linear interpolation between data points is used to determine the speed effect and force effect for operating speed and force. The final correction is calculated by multiplying the interpolated speed effect value times the interpolated per unit force effect.

\subsection{Roll Thermal Expansion}

The work and backup rolls heat up when metal is in the stand and cool down when the stand is empty. As roll temperature increases the diameter expands causing the actual roll gap to decrease.

This effect cannot be measured while rolling so it is modeled by the setup model using the roll material characteristics, metal temperatures, rolling time and length, cooling spray flows, etc. The effect is separated into a long term effect and a short term effect. The long term (pass to pass, product to product) effect is included in a gap position reference offset. The short term effect is modeled as a simple exponential time constant. The setup model provides the Final Value of gap change and the Time Constant for each product.

\subsection{Roll Bending Compensation}

Roll bending is used to help control strip profile and flatness. Depending on the mechanical configuration, the force produced by positive (crown in) roll bending cylinders can be measured by the stand force sensors even though the positive bending forces do not help to reduce the strip. Therefore the strip force is calculated by subtracting the positive roll bending force from the load cell force. Note that the load cells are calibrated to produce zero tons with backup roll balance on, Roll Bending and balance off, and the stand roll gap open that determines a tare force that must also be subtracted from load cell force.

The roll gap position is calibrated with roll bending at a roll balance force. When bending force changes from balance force there will be a change to the effective roll gap. The predominant effect on the roll gap is that an increase in positive bending will stretch the mill housing causing the effective gap to open. MMC AGC will not correct for this stretch because the positive bending force is not included in strip force. The gap correction necessary to compensate for this stretch must close the gap by an amount equal to the bending force (above bending force at gap calibration) divided by the mill housing modulus.

\section{APPLICATIONS}

\subsection{Design Approach}

This thickness control could only be achieved through proper system design. The most important design objectives were high availability, efficient installation, optimum delivery, and ease of maintenance. A flexible modular approach was chosen to enhance these design objectives. This approach was further enhanced with the extensive use of structured design techniques.

Structured design techniques were used throughout both the hardware and software design. The essential nature of structured design involves breaking down the complex requirements into small modules which are then easily managed. The main design effort is involved in the process of building each of these modules and assembling them together to form the complex set of automation functions. Good structured design promotes the flexible modular approach significantly both during the design phase and for the longterm maintenance of the system.

The AGC software was divided into a number of individual control, action, and communication modules. Many of these modules were managed as a coordinated group of AGC functions while other functions were performed as more individual modules separate from this group. The division of the AGC functions is shown in Fig. (6). 


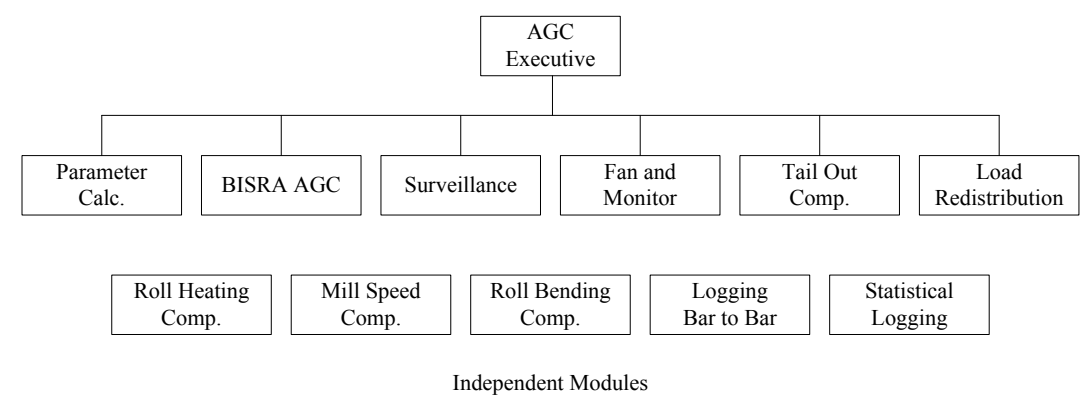

Fig. (6). Software Structure of AGC.

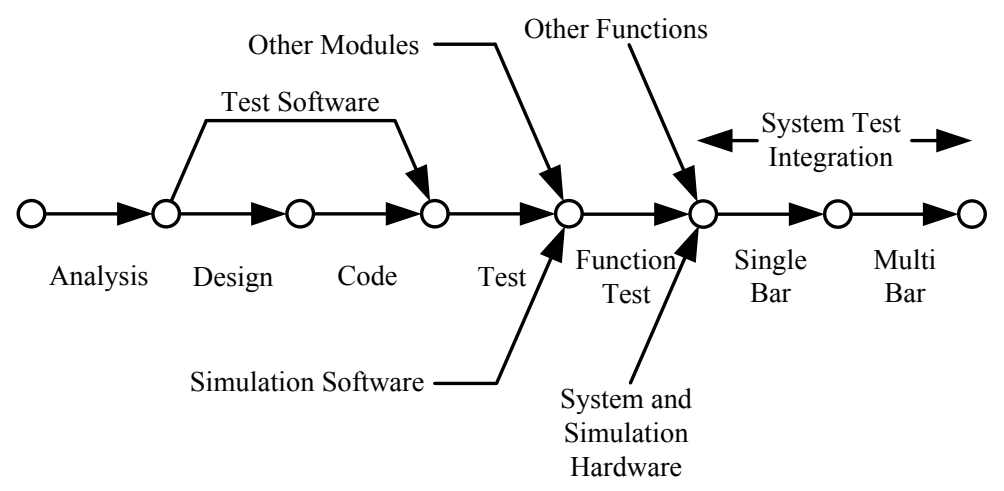

Fig. (7). Software Design Planning Chart.

\subsection{Software Planning}

Each of the individual software modules has a similar life history. Fig. (7) contains a generalized view of that history. There is an analysis phase, a design phase, a coding phase, and an individual testing phase. Groups of modules were brought together to provide functional tests. Then many functions were combined together for a total simulation involving both hardware and software integration. Individual module test software was designed. In addition a very flexible and comprehensive software simulation package was developed and used prior to, and during, the hardware and software integration phase.

In the initial analysis phase fully $80-90$ percent of the final system documentation was produced. This documentation was subject to a rigorous design inspection and review procedure. This procedure was very successful and ensured at an early stage in the project that all the individual parts would eventually fit together correctly. Both customer and factory personnel worked together during the design phase. Work activities were allocated based on skill and experience among all team members.

\section{CONCLUSION}

The plate mill thickness control system described herein was successfully installed at the No. 2 Plate Mill of Handan Hongri Metallurgy Co. Ltd., China. The system has surpassed its original performance targets. This success was not the result of a single factor, but of the thorough coordination of the total thickness control system. This included welldeveloped control strategies, structured design implementa- tion, accurate simulation and calibration of the mill prior to rolling, as well as the availability of fully tested engineering analysis tools for the first day of rolling. The system was quickly brought on-line and has continued to enjoy very stable high performance as a result of these efforts.

The thickness accuracy guaranteed value of the automation system is shown in Table 1. With AGC function in basic automation system, the plate thickness accuracy can be guaranteed.

Table 1. Plate thickness accuracy guaranteed value.

\begin{tabular}{|c|c|c|}
\hline \multirow{7}{*}{$\begin{array}{c}\text { Target Thickness } \\
\text { Range } \\
\mathbf{( m m )}\end{array}$} & $\begin{array}{c}\text { Thickness Tolerance } \\
\mathbf{( 2 \sigma )}\end{array}$ \\
\hline \hline \multirow{4}{*}{$\begin{array}{c}\text { Thm } \\
\text { along one plate } \\
\text { length }\end{array}$} & $6 \leq h \leq 10$ & $\leq 0.08$ \\
\cline { 2 - 3 } & $10<h \leq 20$ & $\leq 0.10$ \\
\cline { 2 - 3 } & $30<h \leq 50$ & $\leq 0.12$ \\
\cline { 2 - 3 } & $50<h$ & $\leq 0.16$ \\
\hline \multirow{4}{*}{$\begin{array}{c}\text { Thickness accuracy } \\
\text { for different plates }\end{array}$} & $6 \leq h \leq 10$ & $\leq 0.24$ \\
\cline { 2 - 3 } & $10<h \leq 20$ & $\leq 0.10$ \\
\cline { 2 - 3 } & $20<h \leq 30$ & $\leq 0.12$ \\
\cline { 2 - 3 } & $30<h \leq 50$ & $\leq 0.15$ \\
\cline { 2 - 3 } & $50<h$ & $\leq 0.20$ \\
\hline
\end{tabular}


We have collected statistics data for 7 months, and conclude from the data analysis that the average thickness qualified rate of the No. 2 plate mill is 1.4 percent higher than the No. 1 Plate Mill which was built two years ago.

Automation of rolling mills is an important and vibrant activity that reaches into every aspect of mill operation and links together a number of academic and commercial disciplines. The role of automation has been well established for a number of years and is likely to expand for many years to come.

\section{CONFLICT OF INTEREST}

The authors confirm that this article content has no conflicts of interest.

\section{ACKNOWLEDGEMENTS}

This work is partially supported by the Fundamental Research Funds for the Central Universities (No. FRF-TP-14104A2) and Beijing Higher Education Young Elite Teacher Project (No. YETP0412).

\section{REFERENCES}

[1] F. Zhang, J. Hou, Q. Yang, Q. Guo, and L. Huang, "Multilevel control of screw-up AGC system of plate mill", Electric Drive, vol. 42, no. 2. pp. 73-77, 2012.
[2] R. Heeg, T. Kiefer, A. Kugi, O. Fichet, and L. Irastorza, "Feedforward Control of Plate Thickness in Reversing Plate Mills", IEEE Transations on industry applications, vol. 43, no. 2, pp. 386-394, 2007..

[3] I. J. Ferguson, and R. F. D. Tina, "Modern Hot-Strip Mill Thickness Control, IEEE Transations on industry applications, vol. IA-22, no. 5, pp.934-940, 1986.

[4] X.N. Wang, and X.K. Ding. "Control system of modern strip rolling mill", Shengyang: Northeastern University Publishing House, 1996.

[5] V. B. Ginzburg. "High-quality steel rolling: theory and practice", New York: Marcel Dekker, Inc, 1993.

[6] Y. Hsu, C. Cheng, C. Chen and L. Hsu. "Development of a Simulator of Automatic Gauge Control System for Hot Strip Mill". Proceedings of the 2002 International Conference on Control and Automation, 2002. pp. 1284-1288

[7] S. Tan, and J. Liu. "Improvement and application of automatic gauge control system in hot strip rolling mills". Proceedings of the 7th World Congress on Intelligent Control and Automation, Jun. 2008. pp. 710-713

[8] G. Rath. "Model based thickness control of the cold strip rolling process", D.S. thesis, University of Leoben, Austria, 2000.

Received: September 16, 2014

Revised: December 23, 2014

Accepted: December 31, 2014

(C) Zhang et al.; Licensee Bentham Open.

This is an open access article licensed under the terms of the Creative Commons Attribution Non-Commercial License (http://creativecommons.org/licenses/by-nc/3.0/) which permits unrestricted, non-commercial use, distribution and reproduction in any medium, provided the work is properly cited. 